\title{
The impact of sedentary and physical activity behaviour on frailty in middle-aged and older adults
}

\author{
Dustin Scott Kehler
}

\begin{abstract}
Physical activity and sedentary behaviours are associated with frailty. However, it is unknown if different accumulation patterns of these behaviours are linked with frailty. Four studies were conducted: the first 3 determined if bouts of moderate-vigorous physical activity (MVPA) and patterns of sedentary behaviours were associated with frailty (study 1), and if sex (study 2) and cardiovascular disease (CVD) status (study 3) affected these associations. Study 4 systematically reviewed the evidence to determine if preoperative physical activity and sedentary behaviours were linked to postcardiac surgical outcomes. Studies 1-3 used accelerometer data from the 2003-2004/2005-2006 National Health and Nutrition Examination Survey. Bouted ( $\geq 10 \mathrm{~min}$ ) and sporadic ( $<10 \mathrm{~min}$ ) durations of MVPA were analyzed based on meeting a proportion of the physical activity guidelines of $150 \mathrm{~min} /$ week. Prolonged sedentary behaviours were measured in bouts lasting $\geq 30 \mathrm{~min}$. Breaks from sedentary behaviour were any $\geq 1$ min interruption in sedentary time. Average intensity and duration during breaks were analyzed. Frailty was measured with a 46-item frailty index (FI). Study 4 included investigations that linked preoperative physical activity behaviours to postoperative health outcomes. The first 3 studies revealed that sporadic and bouted MVPA were associated with a lower FI. Meeting 1\%-49\% of the physical activity guidelines had a protective association with frailty. Prolonged sedentary bouts had a more detrimental association with frailty in females than males. Bouted MVPA was associated with a lower FI in CVD participants but not in those without CVD. Average break intensity was associated with a lower FI across studies. Average break duration was associated with frailty in males and in those with CVD. Study 4 included 11 articles, which reported inconsistent findings in relation to self-reported physical activity behaviour and postoperative outcomes in cardiac surgery patients. No studies analyzed sedentary behaviour or frailty. Data from this thesis suggest that bouts of MVPA and patterns of sedentary behaviours are associated with frailty, and support the need to limit extended periods of sedentary time and promote a physically active lifestyle. Studies are needed to determine if preoperative physical activity and sedentary behaviours are associated with postcardiac surgical frailty.
\end{abstract}

Received 15 February 2018. Accepted 20 February 2018.

D.S. Kehler.*,+, Health, Leisure \& Human Performance Research Institute, Faculty of Kinesiology and Recreation Management, University of Manitoba, Winnipeg, MB R3T 2N2, Canada.

Email for correspondence: d_scott_k@hotmail.com.

*Present address: Geriatric Medicine Research Unit, Division of Geriatric Medicine, Faculty of Medicine, Dalhousie University, Halifax, NS B3H 2E1, Canada. †Advisor: Todd A. Duhamel. Health, Leisure \& Human Performance Research Institute, Faculty of Kinesiology and Recreation Management, 208 Active Living Centre, University of Manitoba, Winnipeg, MB R3T 2N2, Canada.

‡Dr. Kehler is a Postdoctoral Fellow in the Department of Medicine, Division of Geriatric Medicine, under the supervision of Drs. Kenneth Rockwood and Olga Theou. He is pursuing multiple research projects related to aging, frailty, and other geriatric-related health outcomes in relation to sedentary and physical activity behaviours. For example, he holds a Canadian Institutes for Health Research Fellowship for his proposed research work in frailty and cardiac rehabilitation and the influence of sedentary and physical activity behaviours.

Copyright remains with the author(s) or their institution(s). Permission for reuse (free in most cases) can be obtained from RightsLink. 